\title{
Persistent Trigeminal Artery: A Case Report
}

\author{
Vipasha Singh ${ }^{1}$, Sarita Kumari ${ }^{2}$, Jai Choudhary ${ }^{3}$, Hemant Kumar Mishra ${ }^{4}$
}

\begin{abstract}
Persistence of fetal vessel in adulthood, joining the carotid and vertebrobasilar systems in fetal period apart from the posterior communicating artery, is rare. ${ }^{1}$ This communication is called carotid-vertebrobasilar anastomosis, which includes trigeminal, otic, hypoglossal, and proatlantal arteries. A case of a persistent trigeminal artery (PTA) with carotid-vertebrobasilar junction anomaly is found in a young male is reported. Here, PTA arises from cavernous portion of the right inferior cerebellar artery and traveled posteriorly to join basilar artery and terminates and corresponds to TA type II of Saltzman's classification. It is also associated with basilar invagination and cervical cord myelomalacia.

Keywords: Carotid-vertebrobasilar system, Cervical cord myelomalacia, Trigeminal artery.

Journal of Mahatma Gandhi University of Medical Sciences \& Technology (2019): 10.5005/jp-journals-10057-0097
\end{abstract}

\section{INTRODUCTION}

Anastomoses between the carotid and the vertebrobasilar circulations can occur via persistent primitive trigeminal, otic, hypoglossal, and proatlantal arteries. The trigeminal artery (TA) is the most commonly reported among these, and it is named as TA due to its proximity to the trigeminal ganglion. TAs are usually associated with Moyamoya disease, intracranial arteriovenous malformations, and intracranial aneurysms. Persistent TA (PTA) originates from cavernous portion of inferior cerebellar artery (ICA), and its course is defined with its relation to sella turcica, either PTA penetrates sella near clivus or it courses lateral to sella turcica and then terminates in BA. It has variable termination, i.e., it can directly terminate between the anterior ICA (AICA) and the superior cerebellar artery (SCA), or directly on the SCA, or less frequently on the posterior ICA.

\section{Case Description}

A 14-year-old boy was admitted in our hospital on February 2019 with complaints of progressive restricted neck movements and abnormal neck movement since childhood along with progressive weakness in all four limbs since 1 month. He also had difficulty in walking. He had three to four episodes of headache and dizziness in the last 1 month. Magnetic resonance imaging (MRI) of the outer brain was normal; however, MRI of the cervical spine was suggestive of basilar invagination and myelomalacic changes from $\mathrm{C} 1$ to $\mathrm{C} 6$ vertebral levels. On examination, power in bilateral upper limb was $3 / 5$ and in lower limb was $4 / 5$ along with decreased sensation below $\mathrm{C} 4$ dermatome. However, bowel and bladder habits were normal. Computed tomography angiography of th brain and neck was performed which suggested hypoplastic vertebral arteries and proximal basilar artery with abnormal communicating vessel between intracavernous portion of right ICA and distal basilar artery suggestive of persistent primitive trigeminal artery on right side.

\section{Discussion}

Embryology of cerebral arteries helps in understanding the functional significance and anatomy of carotid-vertebro-basilar anastomosis. At 4-mm embryo stage, the supply of forebrain is by ICA, while hindbrain is supplied by the basilar artery formed by
${ }^{1-4}$ Department of Radiodiagnosis, Mahatma Gandhi Medical College and Hospital, Jaipur, Rajasthan, India

Corresponding Author:Vipasha Singh, Department of Radiodiagnosis, Mahatma Gandhi Medical College and Hospital, Jaipur, Rajasthan, India, Phone: +91 9462716623, e-mail: singhvipasha.gurjar@gmail.com How to cite this article: Singh V, Kumari S, Choudhary J, et al. Persistent Trigeminal Artery: A Case Report. J Mahatma Gandhi Univ Med Sci Tech 2019;4(2):46-48.

Source of support: Nil

Conflict of interest: None

longitudinal neural arteries. Carotid arteries supply the longitudinal arteries via TA, otic artery, hypoglossal artery, etc. ${ }^{1}$

At 6-mm stage, the phenomenon of anastomosis between distal ICA and corresponding longitudinal neural arteries occur which further forms the posterior communicating artery. ${ }^{1}$ Subsequently, they regress and obliterate. If there is failure of regression and obliteration of presegmental arteries and proatlantal artery, anastomosis persists in adult. PTA is the fetal

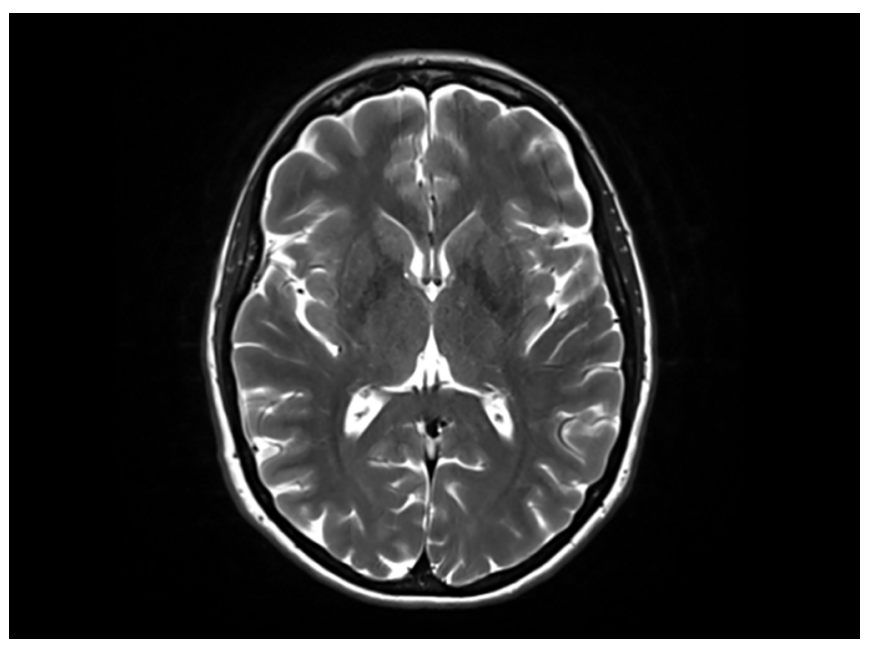

Fig. 1: T2-weighted (T2W) image of brain showing no significant abnormality

(c) The Author(s). 2019 Open Access This article is distributed under the terms of the Creative Commons Attribution 4.0 International License (https://creativecommons. org/licenses/by-nc/4.0/), which permits unrestricted use, distribution, and non-commercial reproduction in any medium, provided you give appropriate credit to the original author(s) and the source, provide a link to the Creative Commons license, and indicate if changes were made. The Creative Commons Public Domain Dedication waiver (http://creativecommons.org/publicdomain/zero/1.0/) applies to the data made available in this article, unless otherwise stated. 

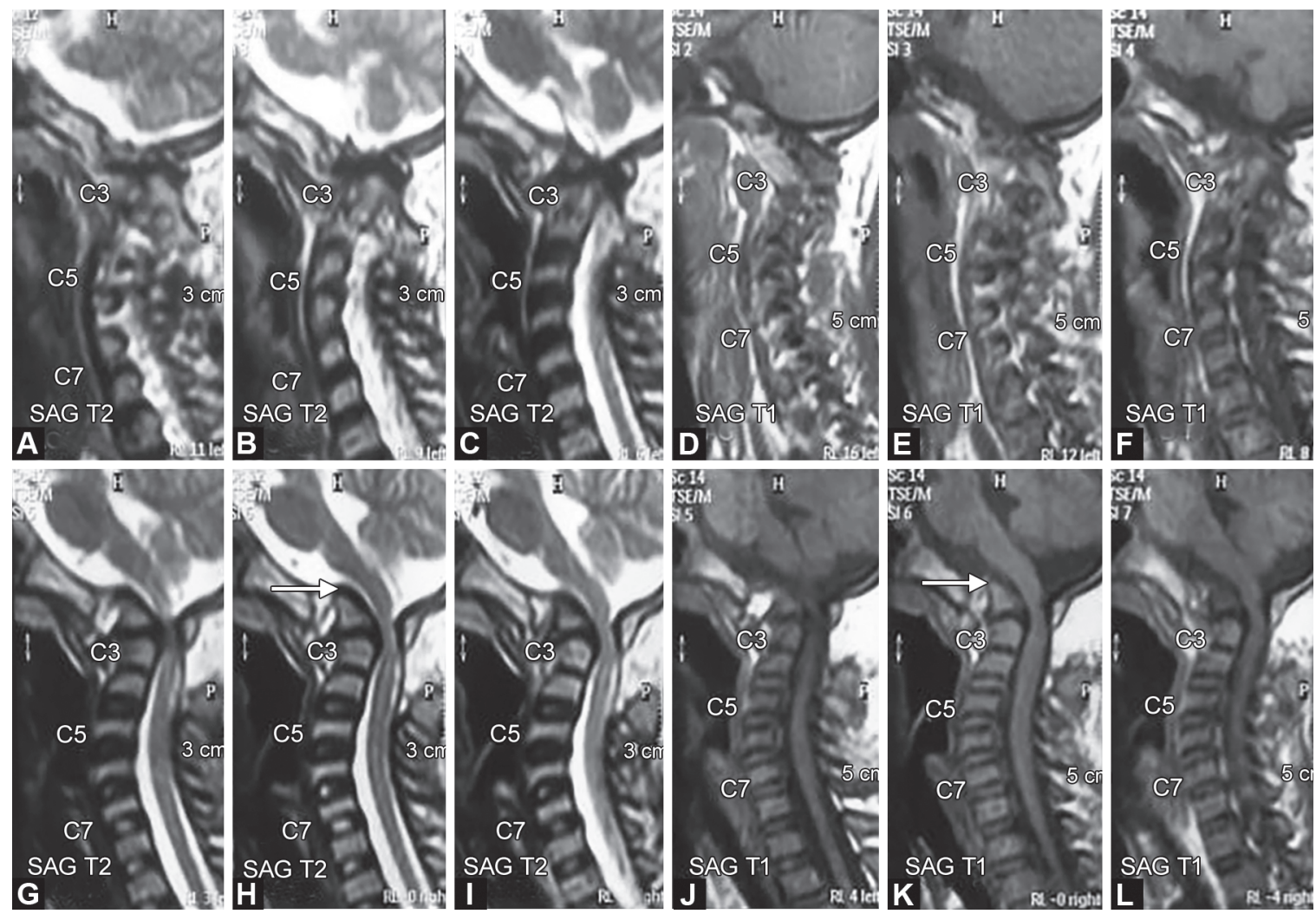

Figs 2A to L: Sagittal T1 and T2 images showing narrowing at C1 level causing T2 hyperintensities from C1 to C6 level (myelomalacia)
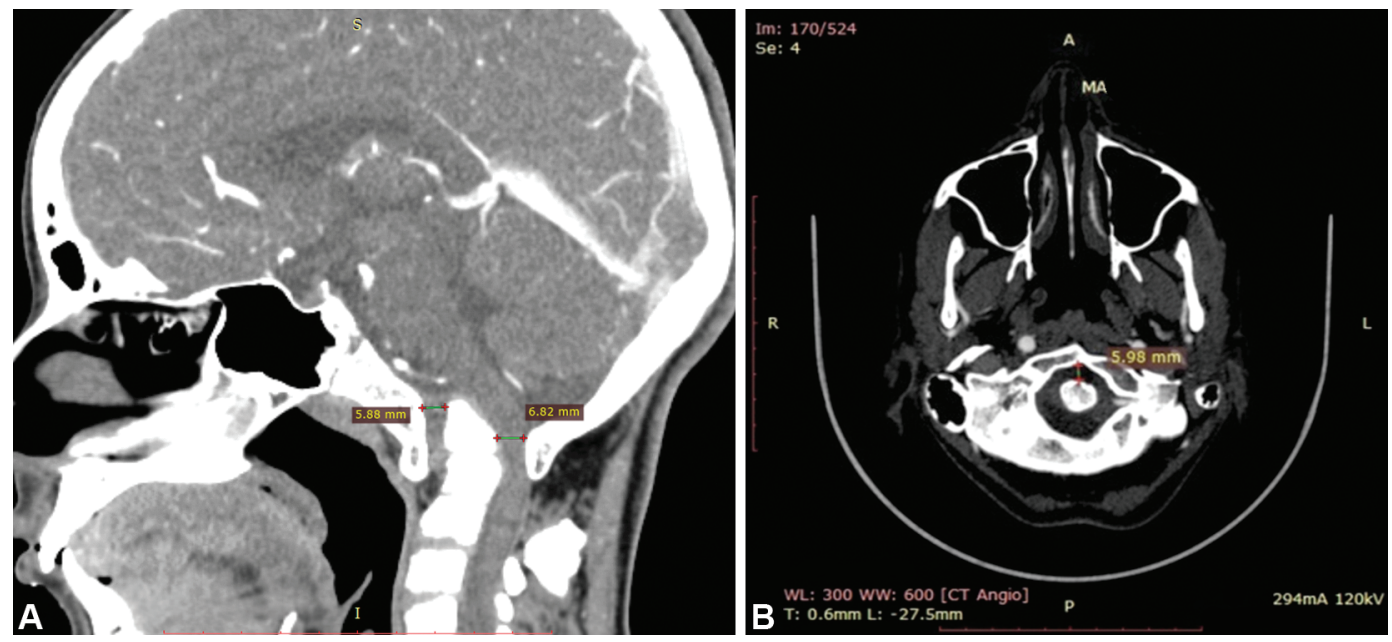

Figs $3 \mathrm{~A}$ and B: Sagittal image of CV junction showing basilar invagination, narrowing at spinal canal at C1 level (AP diameter -6.5 mm), increased atlanto-axial joint space- $5.98 \mathrm{~mm}$

artery supplying the brain stem. TA is last to regress during development. The incidence is $0.2 \%$; however, if we include unreported cases, the incidence is $1 \%$. TA is the most common anomaly (85\%) of all carotid-basilar anastomoses. ${ }^{2-4}$ According to Salas et al.'s classification, there are two types of TA based on relationships with the abducent nerve: a lateral or petrosal and medial or sphenoidal. Classification according to angiographic studies is based on TA termination. According to Saltzman's type I, TA terminates between SCA and AICA and is usually associated with hypoplastic proximal basilar artery. ${ }^{5}$ Absence or poor opacification of posterior communicating arteries is seen. The supply of posterior cerebral arteries and SCA is via TA. According to Saltzman type II, TA supplies the superior cerebellar arteries and terminates between SCA and AICA. The posterior cerebral arteries are supplied by posterior communicating arteries. ${ }^{6}$ TA may terminate in cerebellar artery directly, which sometimes is referred as Saltzman type III, a rare variant. ${ }^{1}$ According to this classification, TA we studied corresponds to Saltzman type II.

\section{Conclusion}

The angiographic finding is that TA is Saltzman's type II. 

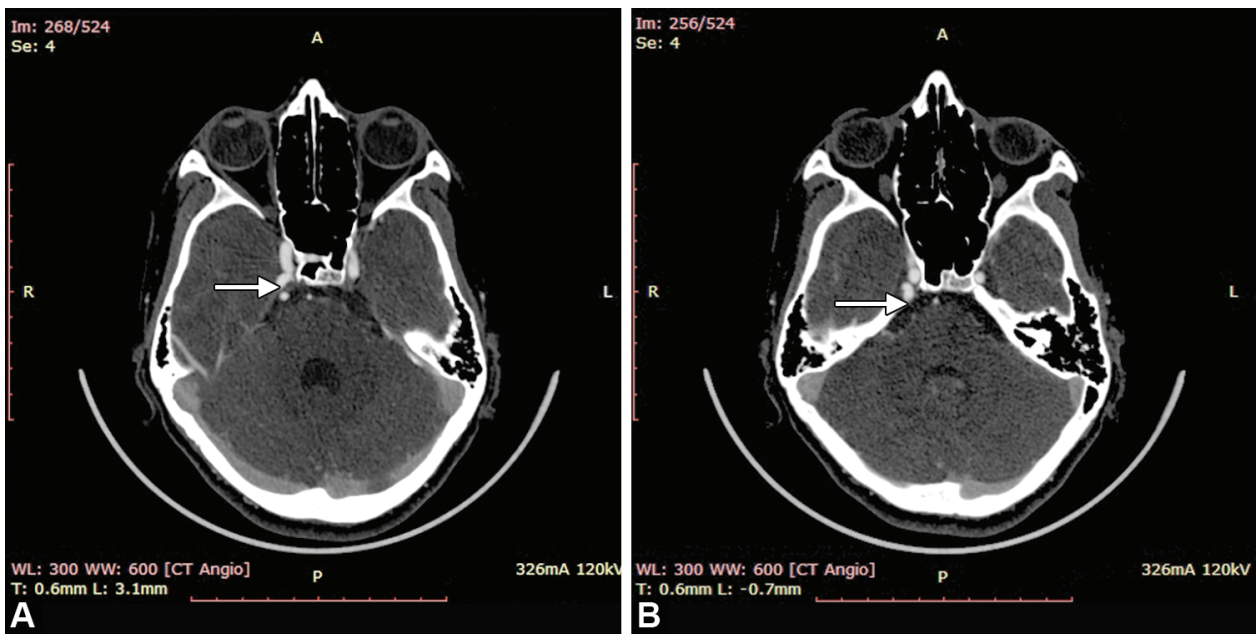

Figs 4A and B: Axial sections of CTA showing abnormal connection between ICA and basilar artery, i.e. persistent trigeminal artery, PTA (arrow)
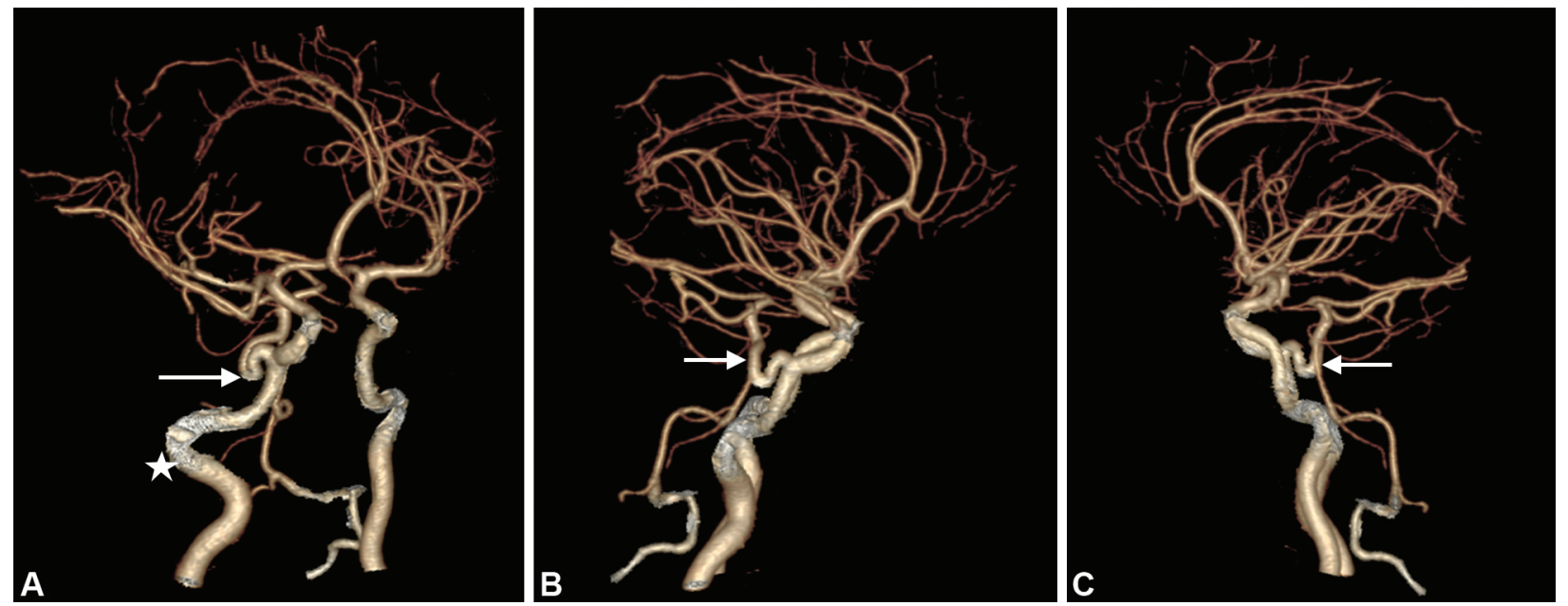

Figs 5 A to C: Showing abnormal communicating vessel (arrow) between intracavernous portion of right ICA (star) and distal basilar artery (normal caliber). B/L vertebral artery and proximal basilar artery-hypoplastic

\section{References}

1. Russo AM, Sienra MC. Persistent trigeminal artery: case report, Departamento de Anatomía, Prof. Dr. Víctor Soria, Facultad de Medicina, Universidad de la República, Montevideo, Uruguay.

2. Meckel S, Spittau B, McAuliffe W. The persistent trigeminal artery: development, imaging anatomy, variants, and associated vascular pathologies. Neuroradiology 2013;55(1):5-16. DOI: 10.1007/s00234011-0995-3.

3. Osborn AG. Osborn's Brain: Imaging, Pathology and Anatomy. 2013. pp. 172-174.

4. Saltzman GF. Patient primitive trigeminal artery studied by cerebral angiography. Acta Radiol 1959;51(5):329-336. DOI: 10.3109/00016925909171103.

5. Lam JJH, Mohamed Shah MTB, Chung SL. Persistent primitive trigeminal artery associated with a cavernous carotid aneurysm. Case report and literature review. J Radiol Case Rep 2018;12(11):1-11. DOI: 10.3941/jrcr.v12i11.3500.

6. Alcalá-Cerra G, Tubbs R, Niño-Hernández L. Anatomical features and clinical relevance of a persistent trigeminal artery. Surg Neurol Int 2012;3(1):111. DOI: 10.4103/2152-7806.101798. 